\title{
OPEN Nanoscale spatial dependence of avidity in an IgG1 antibody
}

\author{
Agnieszka Jendroszek ${ }^{1,2} \&$ Magnus Kjaergaard ${ }^{1,2,3,4 凶}$
}

Antibodies are secreted proteins that are crucial to recognition of pathogens by the immune system and are also efficient pharmaceuticals. The affinity and specificity of target recognition can increase remarkably through avidity effects, when the antibody can bind a multivalent antigen through more than one epitope simultaneously. A key goal of antibody engineering is thus to optimize avidity, but little is known about the nanoscale spatial dependence of avidity in antibodies. Here, we develop a set of anti-parallel coiled-coils spanning from 7 to $20 \mathrm{~nm}$ and validate their structure using biophysical techniques. We use the coiled-coils to control the spacing between two epitopes, and measure how antigen spacing affects the stability of the bivalent antibody:antigen complex. We find a maximal avidity enhancement at a spacing of $13 \mathrm{~nm}$. In contrast to recent studies, we find the avidity to be relatively insensitive to epitope spacing near the avidity maximum as long as it is within the spatial tolerance of the antibody. We thus only see a twofold variation of avidity in the range from 7 to $20 \mathrm{~nm}$. The coiled-coil systems developed here may prove a useful protein nanocaliper for profiling the spatial tolerance and avidity profile of bispecific antibodies.

Antibodies are proteins secreted by the immune system that detect and neutralize foreign molecules. Antibodies contain variable regions that can be combined to generate an abundance of different sequences, which means that they can recognize almost any other molecule. This ability has made antibodies a valuable research tool in biology and recently a successful class of pharmaceuticals ${ }^{1}$. The most abundant class of antibodies (IgG) may bind two identical epitopes simultaneously if the antigens are multivalent or are closely spaced on a surface. In such cases, the combined affinity may be much greater than that for a monovalent interaction, which is known as avidity $^{2}$. Currently, we know little about how avidity in antibodies depends on the spatial arrangement of epitopes, which prevents the rational use of avidity in antibody engineering. Optimization of avidity and multivalency will be particularly important to bispecific antibodies, where engagement of several epitopes is fundamental to the therapeutic effect.

Antibodies are flexible, multidomain proteins allowing their structure to adopt to many different antigens. A common antibody scaffold is shared among the whole class of molecules (Fig. 1A). For IgG antibodies, the binding regions are located at the variable tips of two Fab moieties that are connected to the Fc moiety via flexible hinges. The hinges vary between IgG sub-types, and contain interchain disulfide bonds that connect the two Fab moieties. The flexible hinges allow considerable internal freedom between the Fc- and the Fab-moieties. In the unbound state, antibodies thus occupy an ensemble of different conformations, where the intra-molecular dimensions reach up to $19 \mathrm{~nm}^{3,4}$. Full-length antibodies rarely crystallize, likely because domain orientation is still dynamic in most complexes ${ }^{5}$. However, rare crystal structures of full-length antibodies suggested that a fully extended hinge (Fig. 1A) would allow a distance up to $9 \mathrm{~nm}$ between the binding site and the first disulfide bond in the hinge. This suggests a total reach for bivalent binding of antibodies of $\sim 18 \mathrm{~nm}^{6}$, which is mirrored by the ensemble occupied in the free state.

Avidity affects both the association and dissociation step of a binding reaction. The association rate increases as the antibody can bind to several sites, which simply increase the association rate-constant by the multiplicity of the reaction. Following the initial association, the other Fab moiety can bind in an intra-molecular reaction called ring-closing (Fig. 1B). Ring-closing occurs intra-molecularly and is thus independent of the concentration. Instead, it depends on the structure of the antibody and antigen, which together define an effective concentration $^{7,8}$. Dissociation from a bivalent target requires simultaneous release of both Fab moieties, and thus depends on the ring-closing equilibrium and the effective concentration. In principle, the avidity of a bivalent interaction could be predicted from the effective concentration of ring-closing ${ }^{9}$. Effective concentrations and avidity has previously been studied using either model systems $s^{7,8}$ or theoretical models ${ }^{10-12}$. Neither can fully

${ }^{1}$ Department of Molecular Biology and Genetics, Aarhus University, Aarhus, Denmark. ${ }^{2}$ The Danish Research Institute for Translational Neuroscience (DANDRITE), Aarhus, Denmark. ${ }^{3}$ Aarhus Institute of Advanced Studies (AIAS), Aarhus, Denmark. ${ }^{4}$ The Center for Proteins in Memory (PROMEMO), Aarhus, Denmark. ${ }^{\square}$ email: magnus@ mbg.au.dk 

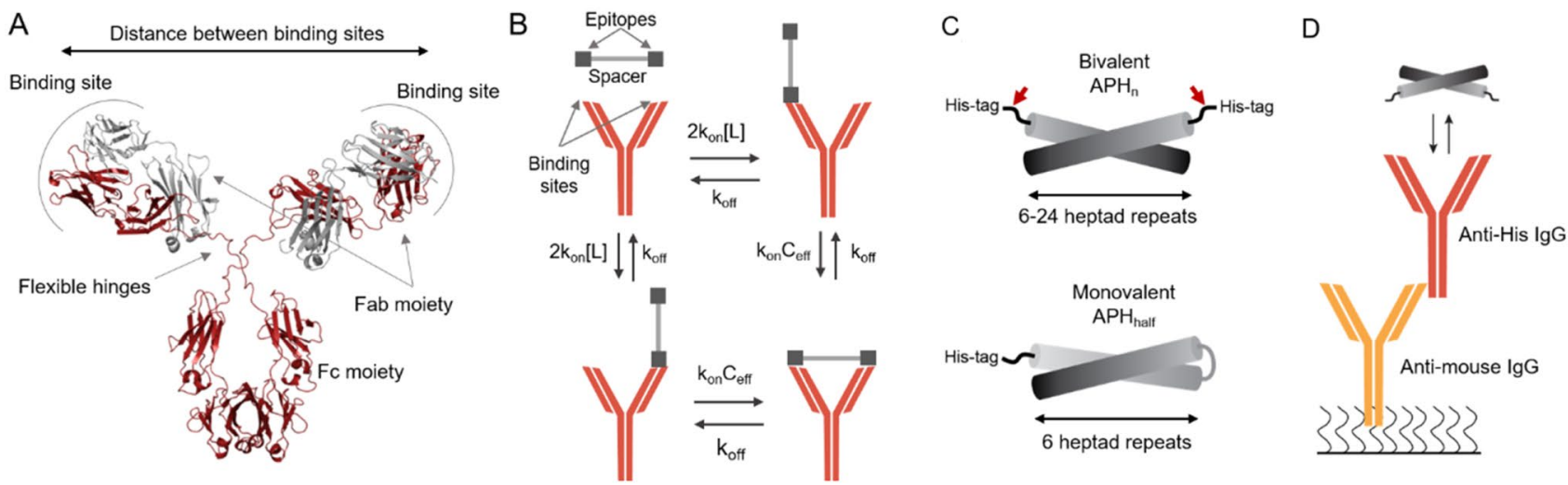

Figure 1. Experimental setup for testing the spatial dependence of avidity in an IgG1 antibody. (A) Domain architecture of an IgG antibody. The flexible hinges allow the two Fab moieties to move independently from the Fc module, and determine the distance tolerated between the two epitopes. (B) Schematic illustration of a bivalent binding between an antibody and an antigen with two epitopes separated by a rigid nanocaliper. The initial association is followed by a an intra-complex ring closing reaction. (C) The design of a bi- and monovalent antigens used to probe the spatial dependence of avidity. The bivalent antigen is composed of two monomers each containing an $\alpha$-helical segment connected to an epitope (His-tag). Helices are composed of six to twenty four heptad repeats and are capable of formation of anti-parallel coiled-coils, which serve as rigid spacers between two epitopes. A thrombin site was introduced between the His-tag and the helix (red arrow) to allow removal of the tag prior to SAXS, DLS and SR-CD experiments. The monovalent antigen is composed of a single His-tag connected to six heptad repeats followed by a flexible loop and six matching heptad repeats, which forms an intramolecular anti-parallel coiled coil. (D) The SPR setup used for avidity measurements includes an anti-mouse antibody targeting the Fc region of the mouse IgG covalently bound to the SPR chip. In each cycle monoclonal mouse anti-His-tag $\mathrm{Ab}$ is captured via interaction with anti-mouse antibody. This allows oriented capture without affecting antibody flexibility and antigen binding.

mimic the complex energy landscape of a dynamic antibody, where bivalent binding will be accompanied by changes in the structural ensemble of the antibody.

The conserved structure of the antibody scaffold suggests that the spatial dependence of avidity may by understood for one antibody and extrapolated to other antibodies in its class. The distance between the epitopes is likely to be one of the principal determinants of avidity. In antibody engineering, it is thus critical to know at which epitope spacing the avidity is the greatest. Similarly, it is critical to determine how large an epitope separation can be tolerated by the antibody, and how strongly avidity depends on the epitope spacing. To answer these questions, we wanted to vary the distance between epitopes in a controlled manner. Inspired by recent successes of DNA nanocalipers ${ }^{13}$ and protein origami ${ }^{14,15}$, we set out to design a set of coiled-coils that could act as nanocalipers between epitopes.

Coiled-coils form rigid extended structures with lengths that depend on the number of heptad repeats. We decided to use anti-parallel coiled-coils, as a linear epitope placed at the end of the helix will result in two identical epitopes at either end (Fig. 1C). The coiled-coils were based on a six-repeat coiled-coil $\left(\mathrm{APH}_{6}\right)$ designed previously ${ }^{16}$, and constructed by concatenation of the $\mathrm{APH}_{6}$ sequence up to 24 heptads in length with additional modifications to prevent out-of-phase dimerization (sequences in the SI). We named the variant with $\mathrm{n}$ heptad repeats $\mathrm{APH}_{\mathrm{n}}$. We also generated a monovalent coiled-coil $\left(\mathrm{APH}_{\text {half }}\right)$ with an identical epitope-presentation by connecting two sequential $\mathrm{APH}_{6}$ sequences with a PGSGSGP-linker to allow formation of an intra-molecular coiled-coil (Fig. 1C). The proteins were expressed in inclusion bodies, and refolded in 3 or 4 steps before purification. The refolding efficiency decreased with increasing length, and for the longest variants was under 5\%, thus preventing the series from being extended further.

The spatial tolerance is determined by the antibody scaffold, and therefore we could choose a convenient epitope as a model system. We used an $\mathrm{N}$-terminal 6xHis-tag connected to the coiled-coil by a short flexible loop (GSS). The His-tag is recognized by several commercial antibodies, which have an affinity that is sensitive to $\mathrm{pH}$ near the $\mathrm{pK}_{\mathrm{a}}$ of histidine. As the antibody structure is independent of $\mathrm{pH}$ from 5.5 to $9^{17}$, this allowed us to tune the monovalent affinity without changing the epitope. The antibody can be uniformly captured on SPR chips using antibodies directed against the Fc moiety of murine IgG antibodies (Fig. 1D). The capture system only recognized the Fc moiety and thus does not affect the two Fab moieties that interact with the antigen.

We characterized the structure of the APH variants by a range of biophysical techniques to test whether they form the desired structures. To isolate the signal from the coiled-coilfrom contributions from the flexible epitope, we used variants with a thrombin cleavable His-tag that only leaves three residues (GSH) before the beginning of the coiled-coil. First, we studied the variants by synchrotron-radiation CD to test whether the proteins form coiled-coils. All CD spectra are characteristic of $\alpha$-helical proteins (Fig. 2A) with DichroWeb ${ }^{18,19}$ suggesting an a-helical fraction of $\sim 90 \%$ for all variants (Table 1). A coiled-coil has a $\mathrm{CD}_{222 \mathrm{~nm}} / \mathrm{CD}_{209 \mathrm{~nm}}$ close to or above $1^{20}$, whereas an isolated helix has a stronger negative peak at $209 \mathrm{~nm}$. All variants have ratios close to 1 consistent with coiled-coil structures. Furthermore, a SR-CD thermal melt of $\mathrm{APH}_{10}$ (Fig. S1) showed a clear sigmoidal 
A

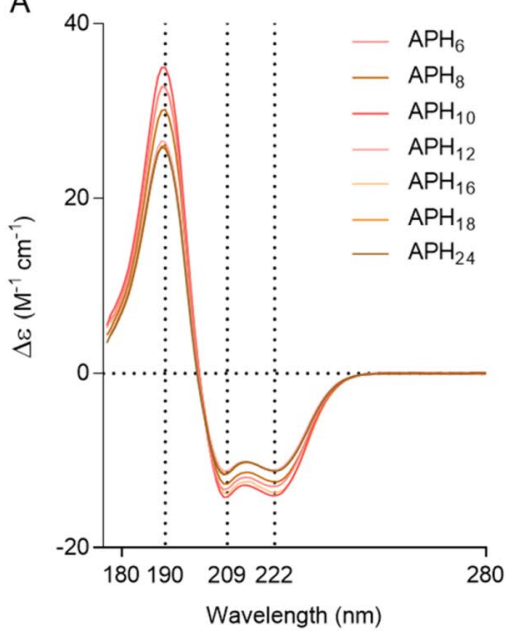

B

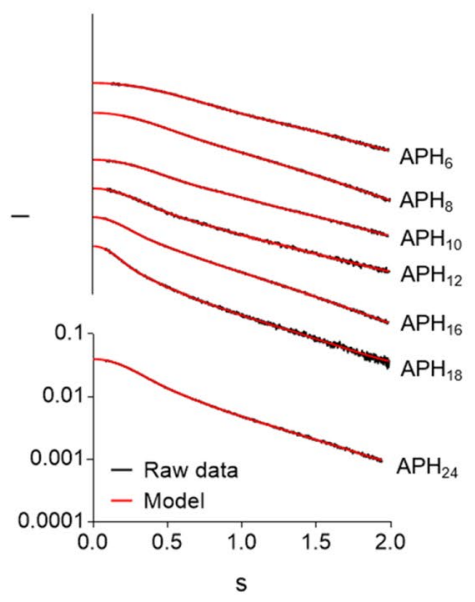

C

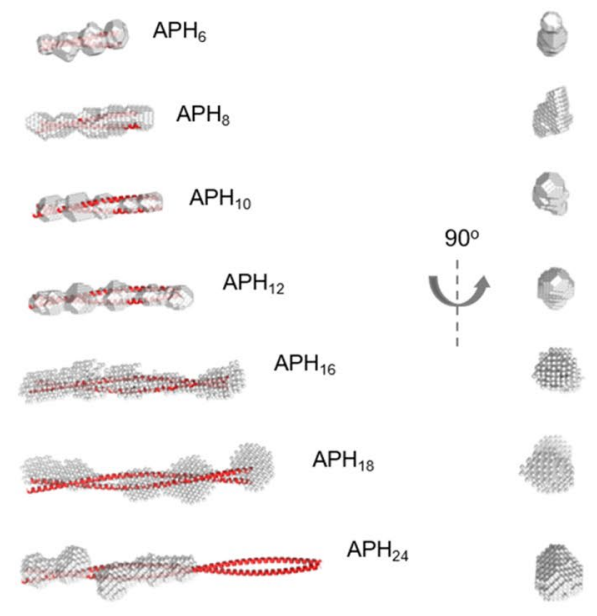

Figure 2. Biophysical characterization of APH variants. Structure of each of the APH spacers was characterized using SR-CD (A) and SAXS (B, C). (A) Shows the SR-CD spectra of spacers with four to twenty four heptad repeats measured at $25^{\circ} \mathrm{C}$. Raw SAXS data (shown in black) and data back predicted from the ab initio models (shown in red) is summarized in (B). Ab initio SAXS models of all APH variants are shown in white with the CCBuilder2.0 models (http://coiledcoils.chm.bris.ac.uk/ccbuilder2/) shown as red helixes (C).

\begin{tabular}{|l|l|l|l|l|l|l|l|l|}
\hline & \multicolumn{3}{|l}{ Synchrotron radiation CD } & \multicolumn{2}{l|}{ SAXS } & DLS & \\
\cline { 2 - 9 } & Helix total & Strand total & Turns & $\boldsymbol{\Delta} \boldsymbol{\varepsilon}_{222}$ to $\Delta \boldsymbol{\varepsilon}_{\mathbf{2 0 9}}$ & Model length $(\mathbf{n m})$ & $\mathbf{R}_{\mathbf{g}}$ & $\mathbf{R}_{\mathbf{h}}$ & Shape factor \\
\hline $\mathrm{APH}_{6}$ & 0.90 & 0.04 & 0.02 & 0.99 & 7.3 & 2.18 & 2.19 & 1.00 \\
\hline $\mathrm{APH}_{8}$ & 0.90 & 0.02 & 0.04 & 0.99 & 10.3 & 2.96 & 2.82 & 1.05 \\
\hline $\mathrm{APH}_{10}$ & 0.89 & 0.05 & 0.02 & 0.99 & 10.6 & 3.00 & 2.70 & 1.11 \\
\hline $\mathrm{APH}_{12}$ & 0.88 & 0.02 & 0.05 & 1.00 & 13.3 & 4.01 & 3.4 & 1.18 \\
\hline $\mathrm{APH}_{16}$ & 0.90 & 0.03 & 0.03 & 1.00 & 17.8 & 4.95 & 4.16 & 1.19 \\
\hline $\mathrm{APH}_{18}$ & 0.91 & 0.03 & 0.03 & 1.00 & 20.2 & 7.57 & 5.34 & 1.42 \\
\hline $\mathrm{APH}_{24}$ & 0.90 & 0.03 & 0.04 & 0.98 & 14.9 & 4.31 & 4.40 & 0.98 \\
\hline
\end{tabular}

Table 1. Biophysical parameters for APH series.

unfolding curve, although with a relatively high degree of helicity in the heat denatured state, suggesting that the coiled-coil unfolded cooperatively.

The overall shape of the APH variants was studied by small angle X-ray scattering (SAXS) (Fig. 2B, S2-3). The scattering curves were used for ab initio model building resulting in elongated shapes for all constructs (Fig. 2C). For coiled-coils containing between 6 and 18 repeats, the lengths of the ab initio shape (Fig. 2C, Table 1) matched idealized coiled-coils constructed using CCBuilder2. $0^{21}$. The cross-section of the ab initio shape-models is roughly uniform through-out the series. This suggests these variants form rigid and stable coiled-coils as desired. The longest variant, $\mathrm{APH}_{24}$, formed a much shorter structure than the intended design. This could suggest an intra-molecular hairpin, but the molecular weight suggests a dimeric form. This variant does not have the intended structure, and will not be discussed in terms of avidity but is included for completeness.

For a more stringent comparison of the SAXS data to the models, we back-predicted the scattering curve from the idealized coiled-coil models using $\mathrm{CRYSOL}^{22}$, and compared it the experimental scattering curve (Fig. S4). APH6 and APH12 show excellent agreement with the back-predicted scattering curve, suggesting that the nanocalipers that their structure is fully described by the idealized model. For $\mathrm{APH}_{8}, \mathrm{APH}_{10}, \mathrm{APH}_{16}$ and $\mathrm{APH}_{18}$ there are notable deviations from the back-predicted curve. This could be due to bending, unfolding, or a minor population of larger complexes. The molecular weight calculated from SAXS data match expectations for a homodimer for $\mathrm{APH}_{6}, \mathrm{APH}_{10}$ and $\mathrm{APH}_{12}$, whereas $\mathrm{APH}_{8}, \mathrm{APH}_{16}$ and $\mathrm{APH}_{18}$ have $16-32 \%$ higher molecular weights than expected. Molecular weights determination by SAXS is less reliable for highly extended molecules ${ }^{23}$, but the molecular weight deviation may indicate larger complexes forming at high protein concentrations. This is especially pronounced for the constructs that are destabilized by removal of repeats from the consensus design such as $\mathrm{APH}_{8}$, or are very long such as $\mathrm{APH}_{16}$ or $\mathrm{APH}_{18}$. Notably, the binding experiments are done at protein concentrations that are at least 100 -fold lower, and do not show any sign of the large avidity effects expected for stable higher order complexes. Thus, it is unlikely that such transient higher order structure have a major impact on the binding experiments. $\mathrm{APH}_{10}$ has the expected molecular weight, but show a small deviation from the predicted scattering for an idealized coiled-coil, however, the ab initio modelling suggested that these structures have the intended average dimensions. This leaves bending of the coiled-coil as the most 
likely explanation, which may broaden the range of permittable epitope spacing, but is unlikely to have a major influence on the average inter-epitope distance.

APH variants were also examined by dynamic light scattering (DLS), which reports on the overall shape and size of the molecule. All samples could be adequately described by a single diffusion time, which suggested that the samples were monodisperse. As expected, the hydrodynamic radius $\left(\mathrm{R}_{\mathrm{h}}\right)$ increased with the number of heptad repeats for all constructs except $\mathrm{APH}_{24}$. The $\mathrm{R}_{\mathrm{g}}$ from SAXS and $\mathrm{R}_{h}$ from DLS can be combined into a shape factor. A sphere is expected to have shape factor of 0.77 , whereas it increases with length for elongated or unfolded molecules ${ }^{24}$. For all APH variants except $\mathrm{APH}_{24}$, the shape factor increased with the number of heptad repeats, consistent with more elongated structures (Table 1). In total, data from SR-CD, SAXS and DLS showed that the APH-variants with between 6 and 18 repeats mainly form the desired rigid, coiled-coil domains.

To estimate the distance between the epitopes, we modelled the flexible His-tags on the idealized coiled-coil nanocalipers using $\mathrm{EOM}^{25}$. The distribution of distances was measured between the third histidine of each tag (Fig. S5). For each nanocaliper, the inter-epitope forms a narrow distribution with a mean that is about a nm longer than the isolated coiled-coil. The successful designs of $\mathrm{APH}_{6}$ to $\mathrm{APH}_{18}$ thus span a mean inter-epitope distance from 8 to $21 \mathrm{~nm}$, which match the expected intra-Fab distances in an antibody. This suggest that these proteins can be used to probe the spatial dependence of avidity by acting as nanocalipers.

As the avidity effect is mainly on the dissociation rate, we used the surface plasmon resonance technique to study the interaction as it allows accurate quantification of tight interactions that vary across orders of magnitude. Furthermore, low-density immobilization of the antibody on a surface prevents formation of higher order complexes. Such higher order complexes are likely to confound analysis of solution experiments. Initial screening of several commercial anti-His-tag antibodies suggested that THE His-antibody from Genscript performed best in terms of affinity and monodispersity. This antibody is a murine IgG1 antibody with native hinge sequences, and can thus be used as a model system for its class. The oriented capture system used here leaves both binding sites exposed for the interaction with antigens (Fig. 1D). Bivalent binding occurs in two steps: An initially monovalent association is followed by an intra-complex ring-closing reaction to form the cyclical complex (Fig. 1B). As SPR measures change in the refractive index corresponding to the mass accumulated on the chip surface, the ringclosing reactions cannot be directly observed. However, dissociation from the antibody require simultaneous dissociation of both sites. When avidity occurs, dissociation is thus slower from bivalent complexes, and can be quantified as the enhancement factor $\beta$ defined by the ratio between $\mathrm{k}_{\text {off_mono }}$ with bivalent $\mathrm{k}_{\text {off }}{ }^{26}$.

To quantify avidity, we measured the interaction kinetics of a monoclonal antibody with mono- and bivalent APH variants. SPR data recorded for $\mathrm{APH}_{\text {half }}$ could be fitted to a 1:1 interaction model (Fig. 3A-C). As expected, the affinity is $\mathrm{pH}$ sensitive and changes from a $\mathrm{K}_{\mathrm{D}}$ of $340 \pm 9 \mathrm{nM}$ at $\mathrm{pH} 5.8$ to $40 \pm 0.2 \mathrm{nM}$ at $\mathrm{pH} 6.2$ (Table S1). The $\mathrm{pH}$ range was not extended further, because lower $\mathrm{pH}$ lead to rapid deterioration of the Fc capture system, whereas high $\mathrm{pH}$ lead to unpractically slow dissociation of bivalent complexes. The dissociation of $\mathrm{APH}_{12} \mathrm{was}$ biphasic at all $\mathrm{pH}$ values (Fig. 3D-F). The rapid component had a dissociation rate similar to that observed for monovalent complexes. This suggested that the fast phase represented proteins bound only to a single site, while the slower phase corresponded to dissociation of bivalently bound protein. The biphasic behavior was more pronounced at higher concentrations, likely because the binding of a second APH dimer to one antibody is concentration-dependent, and thus compete more efficiently with the intra-molecular ring-closing reaction at high concentrations (Fig. S6A). The kinetics could be described well by a global two-component binding model, which resulted in a fast $\mathrm{k}_{\text {off1 } 1}$ similar to $\mathrm{k}_{\text {off_mono }}$ and a slower $\mathrm{k}_{\text {off2 } 2}$. In all case, the fitted second association rate, $\mathrm{k}_{\text {on2 }}$, was vanishingly small (Table S1), which suggested that the system was well-described by a single association. The two-component model used in the BIACORE Evaluation software did not fully capture the concentration dependent amplitudes and produced unrealistically small error estimates. Therefore, we refitted the dissociation rate separately with global rate-constants and variable amplitudes, and used this to estimate the confidence intervals from the $\chi^{2}$-surface (Fig. S7). Both analyses produced similar values (Fig. S7A), but the separate fit of the dissociation phase resulted in a slightly better fit, and these dissociation rates will be discussed in the following.

To map the spatial dependence of avidity, we measured binding kinetics for all bivalent APH variants (Fig. S6). The complete series was measured at $\mathrm{pH} 5.8$, as the $\mathrm{K}_{\mathrm{D}}$ was sufficiently low to allow precise determination of $\mathrm{k}_{\mathrm{off} 2}$ with relatively short dissociation time. All variants showed dissociation traces similar to $\mathrm{APH}_{12}$ (Fig. S6). We recorded the kinetics for $\mathrm{APH}_{24}$, but will not interpret these since they do not form the desired structure. There is no apparent trend in the variation in association rates. The association phase is affected by the silent ring-closing step that is not modelled in the fitting, and we thus do not believe that the association rates are well-constrained by the data. In contrast, the dissociation rates shows a smooth dependence on nanocaliper length. In the following, we analyze the avidity enhancement $\beta$ from complex stability only as defined previously $\left(\beta=\mathrm{k}_{\text {off_mono }} /\right.$ $\left.\mathrm{k}_{\mathrm{off} 2}\right)^{27}$. Comparison of the $\mathrm{k}_{\mathrm{off2} 2}$ to $\mathrm{k}_{\text {off_mono }}$ for all nanocalipers allowed us to determine the avidity enhancement at epitope spacings ranging from 7 to $20 \mathrm{~nm}$ (Fig. $3 \mathrm{G}$ ). The avidity enhancement reached a maximum at a spacing of $13 \mathrm{~nm}$ (30-fold). An increase of the spacing to $20 \mathrm{~nm}$ resulted in a two-fold decrease to a $\beta$ of 12.8 , where as a decrease to $6 \mathrm{~nm}$ lead to a decrease to a $\beta$ of 16.8. This suggested that the spatial variation of avidity was relatively small. While $\beta$-values are intuitively understandable, avidity is best understood in terms of free energy (Fig. $3 \mathrm{H})$. The two-fold difference in $\mathrm{k}_{\text {off }}$ suggests that structural perturbations occurring in the antibody ensemble upon stretching are only on the order of $400 \mathrm{cal} / \mathrm{mol}$. This shows that the energy landscape for avidity is relatively flat for IgG1 antibodies. This suggests that the antibody scaffold has been selected to provide avidity in multivalent antigens with a wide range of spatial arrangements. In total, our results describes a flat energy landscape where the exact distance between antigens has a relatively little influence within the spatial tolerance of the antibody.

During the preparation of this manuscript, two elegant studies appeared that also aimed at describing the spatial dependence of avidity. Using DNA origami to control the spacing of epitopes ${ }^{28}$, Shaw et al. found that maximal avidity occurred at a spacing of $16 \mathrm{~nm}$, and decreased ten-fold at a spacing of $17 \mathrm{~nm}$. This study also suggested that the spatial dependence of avidity depended strongly on the strength of the monovalent affinity ${ }^{28}$. 
A Monovalent antigen $\mathrm{pH} 5.8$

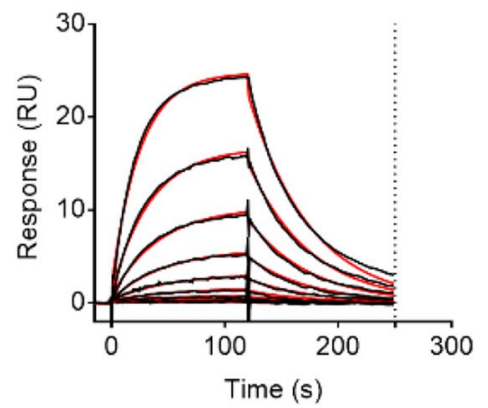

D

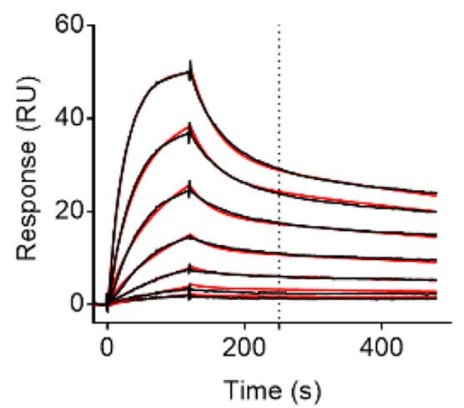

G

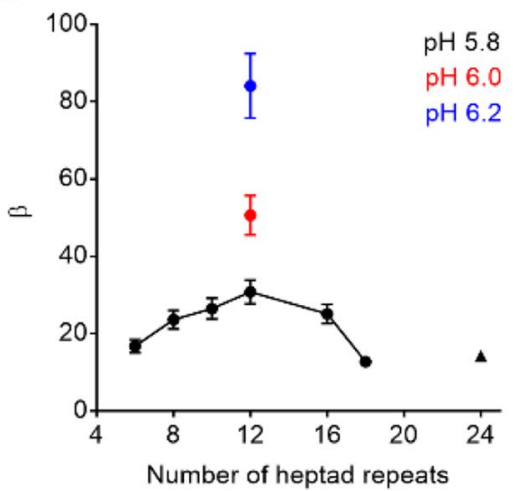

B

Monovalent antigen $\mathrm{pH} 6.0$

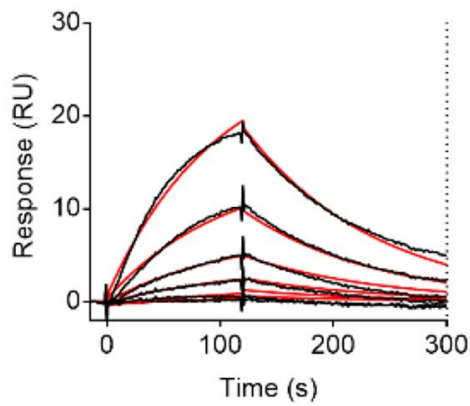

E

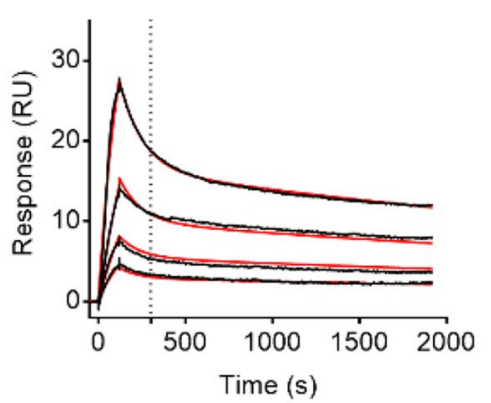

$\mathrm{H}$
C Monovalent antigen $\mathrm{pH} 6.2$

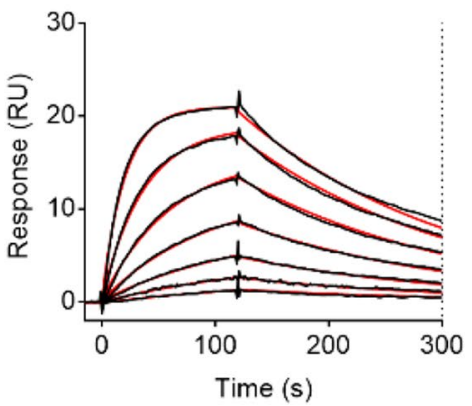

$\mathrm{F}$

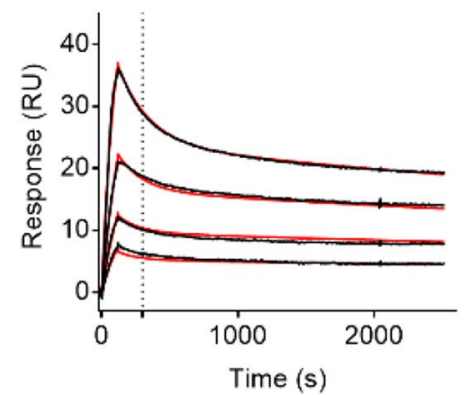

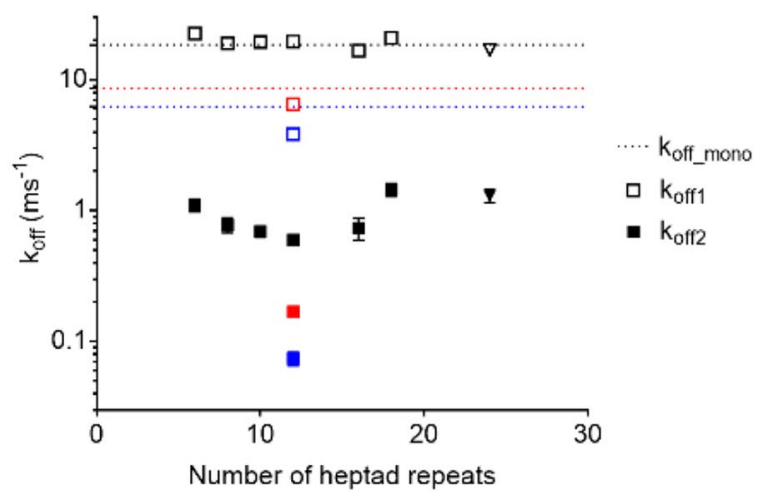

Figure 3. Evaluation of avidity enhancement by surface plasmon resonance. Representative sensorgrams of $\mathrm{APH}_{\text {half }}$ (monovalent) and $\mathrm{APH}_{12}$ (bivalent) binding to the anti-His antibody at various $\mathrm{pH}$ are shown in (A) to (F). $250 \mathrm{~s}(\mathbf{A}, \mathbf{D})$ or $300 \mathrm{~s}(\mathbf{B}, \mathbf{C}, \mathbf{E}, \mathbf{F})$ are marked. Each line represents a single antigen concentration from the dilution series injected over the chip surface. Raw data is shown in black with 1:1 (A, B, C) or bivalent analyte $(\mathrm{D}, \mathrm{E}, \mathrm{F})$ fit shown in red. The bivalent binding produces a biphasic dissociation curve with a fast component $\left(\mathrm{k}_{\mathrm{off} 1}\right)$ coming from the monovalent interaction and slow component $\left(\mathrm{k}_{\mathrm{off} 2}\right)$ coming from the bivalent interaction $\left(\mathrm{k}_{\text {off_bi }}\right.$ ). The affinity enhancement $(\beta)$ expressed as a fold decrease in the $\mathrm{k}_{\text {off_bi }}$ as compared with $\mathrm{k}_{\text {off_mono }}$ determined for monovalent antigen is shown in $(\mathbf{G})$. The $\log \left(\mathrm{k}_{\mathrm{off}}\right)$ values of all antigen variants are summarized in $(\mathbf{H})$ with $\log \left(\mathrm{k}_{\text {off1 } 1}\right)$ and $\log \left(\mathrm{k}_{\text {off } 2}\right)$ are shown as open and full symbols respectively. $\mathrm{k}_{\text {off_mono }}$ is the dissociation rate constant of the monovalent antigen $\left(\mathrm{APH}_{\text {half }}\right)$.

At a monovalent $\mathrm{K}_{\mathrm{D}} \sim 3 \mu \mathrm{M}$, bivalent binding and avidity enhancement was only observed at the optimal epitope spacing, whereas avidity was observed at a broad range of spacings at a monovalent $K_{D} \sim 30 \mathrm{nM}$. Similarly, Zhang et al. used triangular DNA nanostructures and high-speed AFM to measure multivalent binding to epitopes spaced from 3 to $20 \mathrm{~nm}^{29}$. Optimal avidity was observed at a spacing of $10 \mathrm{~nm}$, but with similar values at spacings of 8 and $16 \mathrm{~nm}$, whereas spacings below 5 and above $20 \mathrm{~nm}$ did not give stable bivalent binding. As the goal is to extract a consensus description of avidity in antibodies, it is worthwhile to compare the similarities and differences between these studies.

Our study was designed to take advantage of the $\mathrm{pH}$ dependence of the His-tag binding to tune the monovalent $\mathrm{K}_{\mathrm{D}}$. We have examined the spatial dependence on avidity at $\mathrm{pH} 5.8$, where we see a monovalent $\mathrm{K}_{\mathrm{D}}$ of $320 \mathrm{nM}$. In free energy terms, this is in the middle of the two affinities used by Shaw et al., while Zhang et al. do not report affinities. We observed avidity at a broad range of epitope spacings in agreement with conclusion reached previously for the higher affinity antigen. In principle, the $\mathrm{pH}$ sensitivity of the His-tag should allow us 
to test a wider range of monovalent affinities. In practice, the range of $\mathrm{pH}$ values probed is limited by the stability of the capture system at low $\mathrm{pH}$, and impractical long dissociation times at higher $\mathrm{pH}$. The current design is limited to $\mathrm{pH}$ values ranging from 5.8 to 6.2 , which still results in a threefold decrease in the monovalent $\mathrm{k}_{\text {off }}$. Theory suggests that for a bivalent interaction, the combined affinity is given $b^{30}$ :

$$
K_{D}=\frac{K_{D 1} K_{D 2}}{C_{e f f}}
$$

where $\mathrm{K}_{\mathrm{D} 1}$ and $\mathrm{K}_{\mathrm{D} 2}$ are the monovalent affinities of the two interactions which are identical here, and $C_{e f f}$ is the effective concentration of ring-closing. Alternatively, the strength of a bivalent interaction can be decomposed into twice the free energy of the monovalent interaction, and a "coupling free energy" determined by the molecular architecture ${ }^{17}$. These formulations are equivalent as $C_{e f f}$ determines the coupling free energy. Both formulations thus predict that the avidity enhancement should increase with the strength of the monovalent interaction. In agreement with this, the avidity enhancement increases from 30 -fold to 51 - and 84 -fold (Fig. 3G) at $\mathrm{pH} 6.0$ and 6.2 , respectively. This is roughly consistent with the additivity of the monovalent interaction energies, which predicts that bivalent interaction should have double the $\mathrm{pH}$ dependence of the monovalent in energetic terms. The additivity is not perfect, which is frequently observed in biological interactions due to structural compensation ${ }^{31}$. The assumptions underlying constant "coupling free energy" assumes full ring-closing. It is thus expected that avidity should cease when the effective concentration of the ring-closing falls below the $\mathrm{K}_{\mathrm{D}}$ of the monovalent interaction $^{27}$, which explains why the avidity enhancement eventually ceases at lower monovalent affinities.

In antibody engineering, it is crucial to determine both the spacing providing the maximal avidity enhancement and the total tolerable spacing for bivalent binding. We observe a maximal avidity at a spacing of $13 \mathrm{~nm}$, which is between the optimal spacings reported by Zhang and Shaw et al. of 10 and $16 \mathrm{~nm}$ respectively. Furthermore, we still see bivalent binding with little destabilization at a spacing of $20 \mathrm{~nm}$, whereas Shaw et al. observed at sharp drop in stability at $17 \mathrm{~nm}$ and Zhang does not observe bivalent binding at $20 \mathrm{~nm}$. This discrepancy could be explained if the coiled-coils were slightly bendable and sampled smaller epitope spacings than indicated by the idealized coiled-coil model.

In principle, an ensemble of bent nanocalipers could be fitted to the SAXS curve, however we think this would be overfitting of the data. Instead, we estimated the thermal bending of the coiled-coil based on the general mechanical properties of a dimeric coiled-coil. Previous studies indicate that dimeric coiled-coils can be modelled as a thin filament with a persistence length of $\sim 150 \mathrm{~nm}^{32}$. As the persistence length is much longer than the nanocalipers, they are expected to be relatively straight: A $20 \mathrm{~nm}$ filament with this persistence length is expected to at most bend as an arc-segment of $\sim 28^{\circ}$ through thermal fluctuations. However, this only results in a shortening of the end-to-end distance to $19.5 \mathrm{~nm}$. The strain induced by bivalent binding could in principle cause buckling of the nanocaliper. However, the different in binding affinity between the different nanocalipers provide an upper bound for how much energy is available for deformation. The energy difference of $\sim 400 \mathrm{cal} /$ $\mathrm{mol}$ is less than RT $(593 \mathrm{cal} / \mathrm{mol})$, which suggests that the bending induced by binding to the antibody is less than the thermal fluctuations of the coiled-coil. These approximations suggest that the nanocalipers can bend enough to have an appreciable curvature, but that it has a minimal effect on the epitope spacing.

There are several differences between our protein nanocalipers and a previous DNA origami based studies that could give slightly different distance including the antibodies/antigens, the epitope-linking chemistry, the immobilization strategy and the nature of the nanocaliper. We think the largest contributor is the flexibility of the epitope. An flexible epitope can extend beyond the length of the nanocaliper, or fold back along the coiledcoil. Similarly, it affect the allowed orientations of the bound Fab moiety. Thereby a flexible antigen attachment smoothen the distance dependence and increase the apparent reach of the antibody. The three-residue linker used here only suffice for a tight turn and thus likely represent a minimal flexible connection. An extended sixresidue His-tag is about $2 \mathrm{~nm}$ long, and back-folding of the His-tag towards the center of the coiled-coil would shorten the distance up to $4 \mathrm{~nm}$. In practice, this shortening is likely less than the theoretical maximum due to the strain imposed by binding orthogonal to the coiled-coil. Ensemble simulations in Fig. S5 suggests that APH18 readily accommodates epitope spacings of $18 \mathrm{~nm}$. This suggest that the coiled-coil system developed here likely provides a good estimate of the energetics of antigen spacing near the maximal extension of the antibody. At small epitope spacings, it is likely that the His-tag can be bound in a conformation where they are stretched out from the end of the coiled-coil. Therefore, the size of the epitope itself contributes significantly to the spacer, and it may be inherently difficult to probe the minimal acceptable spacing with flexible epitopes. Therefore, we did not pursue shorter coiled-coil constructs further.

The epitopes used in this study are flexible relative to the nanocaliper, and thus have comparable rotational freedom as the small molecules flexibly conjugated to a surface used previously ${ }^{28,29}$. The rotational freedom of the epitope removes the potential contribution from inter-epitope orientation, which is likely to restrict the range the antibody spans as the Fab fragments have to bind in certain orientations. The distance dependence of fixedangle epitopes is thus likely not well-modeled by the system developed here. However, many multivalent drug targets allow epitopes to rotate relative to each other, for example membrane proteins diffusing in a membrane or protein complexes with multiple domains or subunits. Freely rotating epitopes thus represent a sizeable fraction of antibody targets that are likely to have a similar distance dependence.

The spatial dependence of avidity described here is qualitatively different from that described by Shaw et al. In terms of an energy landscape, Shaw et al. suggest maximal avidity is achieved at the foot of a steep slope, where a slight increase of the distance is very detrimental to binding. We suggest that the optimum avidity is achieved at the bottom of a broad valley, where the precise spacing is not critical as long as it is within the spatial tolerance of the antibody. The latter view was also supported by a recent study which suggested a relatively weak avidity dependence at epitope spacings between 8 and $16 \mathrm{~nm}^{29}$. For the rational use of avidity in antibody 
engineering, this is a critical difference. The energy landscape of avidity is determined by a combination of inter- and intramolecular interactions. Within the spatial range where bivalent interaction can occur without distorting the binding sites, the avidity landscape is thus likely to be dominated by the structural ensemble of the antibody. Structural studies of isolated antibodies suggest that they sample a wide variety of conformations with no particular preferred interdomain orientations. This suggests that most interdomain orientation in antibodies are of similar energy, which is consistent with a wide and flat energy landscape of avidity. For such a flexible bivalent interaction, statistical thermodynamics suggests that optimal avidity should occur when the spacing of the epitopes match the average spacing between binding sites in the flexible antibody ${ }^{10}$. The average spacing is necessarily far from the maximal extension of the antibody. Maximal avidity is thus only likely to occur near the maximal extension of the antibody if new inter or intra-molecular interactions are formed in the bound state. Such interactions have been observed previously and are likely to be system specific ${ }^{5}$. We thus believe the case observed here, where maximal avidity is found at the center of a broad valley represents the more generic case.

The protein nanocaliper system based here has advantages and disadvantages compared to previous systems based on DNA origami. Peptide antigens can readily be encoded into a protein-based system developed here, which is crucial as many antigens are proteins. Furthermore, the spatial resolution of controlled antigen presentation is inherently determined by the pitch of the helix. A coiled-coil based system thus in principle allows controlled spacings down to a single turn of a $\alpha$-helix $(\sim 5 \AA)$, where the local environment surrounding the epitope is identical. The larger turn of a DNA double helix ( $34 \AA$ ) may cause additional contributions from the orientation of the epitope when close epitope spacings are closed. Furthermore, while we do experiments on a surface attached system, the coiled-coil system developed is immediately compatible with solution assays. The main drawback of a coiled-coil based nanocaliper system is the refolding efficiency of the coiled-coil structure decreases strongly with length, likely because the repeating coiled-coil fold is prone to kinetic trapping. Although it would have been desirable to reach a length where bivalent binding is abrogated, the simple design approach we have used here, can thus likely not be extended much further. In contrast, DNA origami can be used to create much larger structures, and much more complex geometric patterns. Both DNA- and protein-based nanocaliper systems have advantages, and we believe that the system developed here represent a useful complement to existing DNA-based technologies.

Avidity holds great promise to enhance the affinity and specificity of target recognition by antibodies. Bivalent binding is particularly important in bispecific antibodies, where Fab moieties that bind different epitopes are combined. In such systems, simultaneous engagement of the epitopes is crucial to the desired therapeutic effect. Likely, nanocaliper based control of the epitope spacing will play a key role in elucidating the spatial and energetic landscape that underlies bivalent binding, and may help develop new antibody formats that optimize avidity.

\section{Materials and methods}

Preparation of DNA constructs. Synthetic DNA of APH constructs were purchased from Gene Universal (Newark, USA) and GenScript (Piscataway, USA) cloned into a pET15b expression vector. The protein variants contained a 6xHis-tag followed by a short flexible liker (GSS) before the N-terminus of the coiled-coil. A thrombin site was introduced between the 6xHis-tag and the coiled-coil using the QuikChange Lightning mutagenesis kit (Agilent, Santa Clara, USA). Sequences of the products were confirmed by DNA sequencing. Sequences of all variants are given in the supplementary material.

Expression, purification and refolding of APH variants. All APH variants were expressed in inclusion bodies in E. coli BL21 (DE3). Cells were cultured over-night at $37^{\circ} \mathrm{C}$ in autoinduction medium ${ }^{33}$ supplied with $100 \mu \mathrm{g} / \mathrm{ml}$ ampicillin and harvested by centrifugation $\left(8983 \mathrm{~g}, 20 \mathrm{~min}, 4^{\circ} \mathrm{C}\right)$. Cell pellets were resuspended in lysis buffer containing $50 \mathrm{mM}$ Tris- $\mathrm{HCl} \mathrm{pH} \mathrm{8.0,200} \mathrm{mM} \mathrm{NaCl}, 1 \mathrm{mM}$ EDTA, $1 \%$ Triton X-100, incubated on ice for approx. $1 \mathrm{~h}$ and disrupted by sonication. Insoluble fraction containing inclusion bodies was then isolated by centrifugation $\left(14,000 \mathrm{~g}, 4^{\circ} \mathrm{C}, 30 \mathrm{~min}\right)$ and washed in $20 \mathrm{mM} \mathrm{Na}_{2} \mathrm{HPO}_{4}, 0.5 \mathrm{M} \mathrm{NaCl}, \mathrm{pH} 7.4$ by gentle stirring for $30 \mathrm{~min}$ at $4{ }^{\circ} \mathrm{C}$ followed by removal of the buffer by centrifugation $\left(14,000 \mathrm{~g}, 30 \mathrm{~min}, 4{ }^{\circ} \mathrm{C}\right)$. Inclusion bodies were solubilized in $20 \mathrm{mM} \mathrm{Na}_{2} \mathrm{HPO}_{4}, 0.5 \mathrm{M} \mathrm{NaCl}, 6 \mathrm{M}$ urea $\mathrm{pH} 7.4$ by over-night gentle stirring at $4{ }^{\circ} \mathrm{C}$ followed by centrifugation $\left(14,000 \mathrm{~g}, 30 \mathrm{~min}, 4^{\circ} \mathrm{C}\right)$ to remove insoluble cell debris. APH variants were purified from urea-resuspended inclusion bodies in the presence of $6 \mathrm{M}$ urea on a gravity Ni-sepharose (GE Healthcare) column and eluted with the step gradient including imidazole concentrations $5 \mathrm{mM}, 20 \mathrm{mM}, 40 \mathrm{mM}, 60 \mathrm{mM}$, $80 \mathrm{mM}, 100 \mathrm{mM}$ and $500 \mathrm{mM}$.

Fractions containing antigens were diluted in buffer containing $6 \mathrm{M}$ urea to a protein concentration of $0.5-1 \mathrm{mg} / \mathrm{ml}$ and refolded by stepwise removal of a denaturation agent. For $\mathrm{APH}_{6}, \mathrm{APH}_{8}, \mathrm{APH}_{10}$ and $\mathrm{APH}_{12}$, refolding included three dialysis steps in $20 \mathrm{mM} \mathrm{Na}_{2} \mathrm{HPO}_{4}, 66.7 \mathrm{mM} \mathrm{NaCl}, \mathrm{pH} 7.4$ at $4{ }^{\circ} \mathrm{C}$ : (I) $3 \mathrm{M}$ urea, overnight. (II) $1 \mathrm{M}$ urea, $\sim 8 \mathrm{~h}$. (III) no urea, overnight. For $\mathrm{APH}_{16}, \mathrm{APH}_{18}$, and $\mathrm{APH}_{24}$, refolding included four dialysis steps (I) $4.5 \mathrm{M}$ urea, overnight. (II) $2.5 \mathrm{M}$ urea, $\sim 8 \mathrm{~h}$. (III) $1 \mathrm{M}$ urea, over-night. (IV) no urea, $\sim 8 \mathrm{~h}$. Misfolded proteins were removed by centrifugation $\left(14,000 \mathrm{~g}, 4^{\circ} \mathrm{C}, 30 \mathrm{~min}\right)$, heating to $80^{\circ} \mathrm{C}$ for $20 \mathrm{~min}$, followed by centrifugation to remove proteins that were not thermostable. Supernatants were purified on the Superdex75 10/300 GL column (GE Healthcare) $\left(\mathrm{APH}_{6}, \mathrm{APH}_{8}, \mathrm{APH}_{10}, \mathrm{APH}_{12}\right)$ or Superdex200 10/300 GL (GE Healthcare) $\left(\mathrm{APH}_{16}, \mathrm{APH}_{18}\right.$, and $\left.\mathrm{APH}_{24}\right)$ equilibrated in $20 \mathrm{mM} \mathrm{Na}_{2} \mathrm{HPO}_{4}, 0.3 \mathrm{M} \mathrm{NaCl}, \mathrm{pH}$ 7.4. Size exclusion of $\mathrm{APH}_{24}$ was additionally followed by ion exchange chromatography on Source S15 4.6/100 PE (GE Healthcare) column in buffer containing $20 \mathrm{mM} \mathrm{Na}_{2} \mathrm{HPO}_{4} \mathrm{pH}$ 7.4. $\mathrm{APH}_{24}$ was eluted with a linear gradient of $0-1 \mathrm{M} \mathrm{NaCl}$ over $10 \mathrm{CV}$. These purification procedures resulted in a protein of more than $95 \%$ purity as judged by SDS-PAGE.

The samples used for SAXS, SR-CD and DLS contained a thrombin-site to allow removal of the His-tag. These proteins were purified identically to the variants without the cleavage site. The purified protein was incubated and 
for $1 \mathrm{~h}$ at $37^{\circ} \mathrm{C}$ with bovine thrombin (Sigma) in a 1000:1 molar ratio. Thrombin was then removed by heating to $80^{\circ} \mathrm{C}$ for $20 \mathrm{~min}$ followed by centrifugation.

Synchrotron radiation circular dichroism (SR-CD). All spacers with removed His-tag were analyzed by SR-CD at AU-CD beam line in ASTRID2 storage ring, Aarhus University, Aarhus, Denmark. In order to remove $\mathrm{Cl}^{-}$ions all spacers were dialyzed against $20 \mathrm{mM} \mathrm{Na}_{2} \mathrm{HPO}_{4}, 0.15 \mathrm{M} \mathrm{NaF}, \mathrm{pH}$ 7.4 prior SR-CD measurements. Concentration of each spacer was brought to approx. $0.7-1 \mathrm{mg} / \mathrm{ml}$ what resulted in absorbance below 1. APH spacers were scanned three times over 280 to $170 \mathrm{~nm}$ at $25^{\circ} \mathrm{C}$ using quartz cuvette with $0.102 \mathrm{~mm}$ pathlength ${ }^{34}$. Reference spectrum from the buffer was obtained analogously and was subtracted from the protein spectrum prior data analysis. For visualization all three protein spectra were averaged and mildly smoothened with a 7 point Savitzky-Golay filter ${ }^{35}$. To compare different linker lengths spectra were scaled according to the protein concentation calculated from the absorption at $205 \mathrm{~nm}$. Secondary structure content was calculated using DichroWeb server (http://dichroweb.cryst.bbk.ac.uk/) ${ }^{18,19}$.

Melting experiment was performed for $\mathrm{APH}_{10}$. Sample preparation and parameters used for measurement were analogical as for analysis at $25^{\circ} \mathrm{C}$. Melting was followed at temperatures from 25 to $85^{\circ} \mathrm{C}$ with $5{ }^{\circ} \mathrm{C}$ steps. After reaching each step system equilibrated for $5 \mathrm{~min}$ after which protein spectrum was measured over 280 to $170 \mathrm{~nm}$. After reaching $85^{\circ} \mathrm{C}$ samples were cooled down to $25^{\circ} \mathrm{C}$ and protein spectrum was measured again.

Small angle X-ray scattering (SAXS). The synchrotron SAXS data was collected at beamline P12 operated by EMBL Hamburg at the PETRA III storage ring (DESY, Hamburg, Germany) ${ }^{36}$. All measurements were performed at $20^{\circ} \mathrm{C}$ in $20 \mathrm{mM} \mathrm{Na}_{2} \mathrm{HPO}_{4}, 150 \mathrm{mM} \mathrm{NaCl} \mathrm{pH} \mathrm{7.4}$. To minimize radiation damage $5 \mathrm{mM}$ DTT was added to each sample and the reference buffer immediately before measurements. Signal from each spacer was obtained for at least three protein concentrations varying from $14 \mathrm{mg} / \mathrm{ml}$ to $0.5 \mathrm{mg} / \mathrm{ml}$. As no indication of the concentration dependent scattering was observed model reconstruction was performed for the highest concentration only where signal to noise ratio was the lowest. Scattering from the buffer was subtracted from the raw data and the scattering curves were brought to absolute scale using known scattering cross section of water. Prior analysis data was averaged and normalized to the intensity of the incident beam. The ab initio models were build using DAMMIF software ${ }^{37}$, a part of the ATSAS 2.8.4. package ${ }^{22}$, using 10 repetitions and P1 symmetry. Models were averaged with DAMAVER ${ }^{38}$ and refined with DAMMIN ${ }^{39}$. Idealized coiled-coil models were built using CCBuilder2.0 (http://coiledcoils.chm.bris.ac.uk/ccbuilder2/) ${ }^{21}$, and the His-tags were modelled as pseudo-atoms in a random coil conformation using $\mathrm{EOM}^{25}$.

Dynamic light scattering (DLS). All experiments were performed on a Wyatt DynaPro NanoStar DLS instrument at $20^{\circ} \mathrm{C}$ on samples from size exclusion chromatography. The data was analysed using the DYNAMICS software to extract the hydrodynamic radius $\left(\mathrm{R}_{\mathrm{h}}\right)$.

Surface plasmon resonance (SPR). Affinity of an interaction between the antigen and antibody were determined using SPR. All measurements were performed on Biacore T200 (GE Healthcare) instrument at $25^{\circ} \mathrm{C}$, flow rate $30 \mu \mathrm{l} / \mathrm{min}$ in $20 \mathrm{mM} \mathrm{Na}_{2} \mathrm{HPO}_{4}, 0.5 \mathrm{M} \mathrm{NaCl}, 0.05 \%$ Tween 20, $0.1 \% \mathrm{BSA}$ at pH 6.2, 6.0 or 5.8. The high $\mathrm{NaCl}$ concentration was necessary to prevent non-specific electrostatic sticking. The SPR chip was prepared by amine coupling of a polyclonal rabbit anti-mouse IgG (GE Healthcare) on both flow cells of CMD500 chip (Xantec). Immobilization was conducted according to the protocol described by manufacturer and resulted in capture of approx. $8000 \mathrm{RU}$ on both active and reference flow cell. Polyclonal anti-mouse IgG targets Fc region of mouse IgG enabling oriented capture of mouse IgG with both Fab moieties exposed for the antigen binding. To determine the binding affinities between antigens and antibody mouse monoclonal anti-His-tag IgG (clone 6G2A9, The His tag Ab, GenScript) was captured on the surface of active flow cell to the level of 100-200 RU. All antigens with various spacer length were tested for binding at $\mathrm{pH}$ 5.8. In each cycle single concentration of an antigen from twofold dilution series (200-1.6 $\mathrm{nM}$ for bivalent and 800-3.2 $\mathrm{nM}$ for monovalent antigen) was injected over both flow cells for $120 \mathrm{~s}$ allowing association. Dissociation was then measured for $360 \mathrm{~s}$ of constant buffer injection. Between the cycles chip surface was fully regenerated by three $15 \mathrm{~s}$ injections of low $\mathrm{pH}$ buffer containing $10 \mathrm{mM}$ glycine $\mathrm{pH}$ 1.7.

Non-specific binding was removed from the raw data by subtraction of signal from the parallel experiment performed in the reference flow cell without anti-His-tag antibody. Buffer injection was also subtracted from the raw data (i.e. double buffer referencing). Data was analyzed in Biacore T200 Evaluation Software (GE Healthcare). For antigens forming monovalent interactions $\left(\mathrm{APH}_{\text {half }}\right.$ and $\left.\mathrm{APH}_{4}\right)$ kinetic constants were determined by fitting 1:1 Langmuir interaction model to the binding curves. For antigens bivalently interacting with an antibody $\left(\mathrm{APH}_{6}, \mathrm{APH}_{8}, \mathrm{APH}_{10}, \mathrm{APH}_{12}, \mathrm{APH}_{16}, \mathrm{APH}_{18}\right.$, and $\left.\mathrm{APH}_{24}\right)$ bivalent analyte model was fitted to the binding curves using one global set of kinetic parameters. Dissociation of these antigens was biphasic with the fast phase more pronounced at high concentrations. The fast phase likely results from two APH dimers bound monovalently to the same antibody, whereas the slow phase refers to dissociation of bivalent antigen:antibody complex (Figure S2). Representative sensorgrams with the fit are shown in (Figure S2). As the concentration dependence of two phases is not included in any of the Biacore Evaluation models, we fitted the dissociation phase globally to a biexponential decay with shared kinetics rates, but variable amplitudes in IGOR Pro (Wavemetrics). The 95\%-confidence interval was estimated from the global fit as $\chi^{2}{ }_{\min } / \chi^{2}>0.9^{40}$. Fitted parameters are summarized in (Table S1).

For high affinity complexes, i.e. $\mathrm{APH}_{12}$ at $\mathrm{pH} 6.0$ and $\mathrm{pH}$ 6.2, $360 \mathrm{~s}$ were not enough to observe biphasic dissociation and decrease in signal sufficient for $\mathrm{k}_{\text {off }}$ determination. To precisely determine dissociation rate of those complexes antigen was constantly injected over both flow cells for $120 \mathrm{~s}$ and then buffer was constantly injected for $30 \mathrm{~min}$ or $40 \mathrm{~min}$. Representative sensorgrams with the fit are shown in Fig. 3. 
Estimation of bending of coiled-coils. The bending of the nanocalipers was modelled based on the energetics of a thin linear filament following a circular arc. The filament is characterized by a persistence length $\left(\mathrm{L}_{\mathrm{p}}\right)$ and a total contour length $\left(\mathrm{L}_{\mathrm{c}}\right)$, and the average angle of bending $\langle\theta\rangle$ and resulting end-to-end distance $<r_{\text {ee }}>$ can be estimated from ${ }^{41}$ :

$$
\begin{gathered}
\left\langle\theta^{2}\right\rangle=2 L_{c} / L_{p} \\
\left\langle r_{e e}^{2}\right\rangle=2 L_{p} L_{c}-2 L_{p}^{2}\left(1-\exp \left(-\frac{L_{c}}{L_{p}}\right)\right)
\end{gathered}
$$

The persistence length was estimated at $150 \mathrm{~nm}$ based on previous studies of the energetics of bending of dimeric coiled-coils ${ }^{32}$.

Received: 19 October 2020; Accepted: 4 June 2021

Published online: 16 June 2021

\section{References}

1. Ecker, D. M., Jones, S. D. \& Levine, H. L. The therapeutic monoclonal antibody market. $m A b s$ 7(1), 9-14. https://doi.org/10.4161/ 19420862.2015.989042 (2015).

2. Vorup-Jensen, T. On the roles of polyvalent binding in immune recognition: Perspectives in the nanoscience of immunology and the immune response to nanomedicines. Adv. Drug Deliv. Rev. 64(15), 1759-1781. https://doi.org/10.1016/j.addr.2012.06.003 (2012).

3. Zhang, X. et al. 3D structural fluctuation of IgG1 antibody revealed by individual particle electron tomography. Sci. Rep. 5(1), 09803. https://doi.org/10.1038/srep09803 (2015).

4. Tian, X. et al. In-depth analysis of subclass-specific conformational preferences of IgG antibodies. IUCrJ 2(1), 9-18. https://doi. org/10.1107/S205225251402209X (2015).

5. Jay, J. et al. IgG antibody 3D structures and dynamics. Antibodies 7(2), 18. https://doi.org/10.3390/antib7020018 (2018).

6. Saphire, E. O. et al. Contrasting IgG structures reveal extreme asymmetry and flexibility. J. Mol. Biol. 319(1), 9-18. https://doi.org/ 10.1016/S0022-2836(02)00244-9 (2002).

7. Mack, E. T., Snyder, P. W., Perez-Castillejos, R. \& Whitesides, G. M. Using covalent dimers of human carbonic anhydrase II to model bivalency in immunoglobulins. J. Am. Chem. Soc. 133(30), 11701-11715. https://doi.org/10.1021/ja2038084 (2011).

8. Mack, E. T. et al. Dependence of avidity on linker length for a bivalent ligand-bivalent receptor model system. J. Am. Chem. Soc. 134(1), 333-345. https://doi.org/10.1021/ja2073033 (2012).

9. Bobrovnik, S. A. The influence of rigid or flexible linkage between two ligands on the effective affinity and avidity for reversible interactions with bivalent receptors. J. Mol. Recognit. 20(4), 253-262. https://doi.org/10.1002/jmr.836 (2007).

10. Diestler, D. J. \& Knapp, E. W. Statistical thermodynamics of the stability of multivalent ligand-receptor complexes. Phys. Rev. Lett. 100(17), 178101. https://doi.org/10.1103/PhysRevLett.100.178101 (2008).

11. Diestler, D. J. \& Knapp, E. W. Statistical mechanics of the stability of multivalent ligand-receptor complexes. J. Phys. Chem. C 114(12), 5287-5304. https://doi.org/10.1021/jp904258c (2010).

12. Numata, J., Juneja, A., Diestler, D. J. \& Knapp, E.-W. Influence of spacer-receptor interactions on the stability of bivalent ligandreceptor complexes. J. Phys. Chem. B 116(8), 2595-2604. https://doi.org/10.1021/jp211383s (2012).

13. Shaw, A. et al. Spatial control of membrane receptor function using ligand nanocalipers. Nat. Methods 11(8), 841-846. https://doi. org/10.1038/nmeth.3025 (2014).

14. Ljubetič, A. et al. Design of coiled-coil protein-origami cages that self-assemble in vitro and in vivo. Nat. Biotechnol. https://doi. org/10.1038/nbt.3994 (2017).

15. Lapenta, F., Aupič, J., Strmšek, Ž \& Jerala, R. Coiled coil protein origami: From modular design principles towards biotechnological applications. Chem. Soc. Rev. 47(10), 3530-3542. https://doi.org/10.1039/C7CS00822H (2018).

16. Gurnon, D. G., Whitaker, J. A. \& Oakley, M. G. Design and characterization of a homodimeric antiparallel coiled coil. J. Am. Chem. Soc. 125(25), 7518-7519. https://doi.org/10.1021/ja0357590 (2003).

17. Tian, X., Langkilde, A. E., Thorolfsson, M., Rasmussen, H. B. \& Vestergaard, B. Small-angle X-ray scattering screening complements conventional biophysical analysis: Comparative structural and biophysical analysis of monoclonal antibodies IgG1, IgG2, and IgG4. J. Pharm. Sci. 103(6), 1701-1710. https://doi.org/10.1002/jps.23964 (2014).

18. Whitmore, L. \& Wallace, B. A. Protein secondary structure analyses from circular dichroism spectroscopy: Methods and reference databases. Biopolymers 89(5), 392-400. https://doi.org/10.1002/bip.20853 (2008).

19. Whitmore, L. \& Wallace, B. A. DICHROWEB, an online server for protein secondary structure analyses from circular dichroism spectroscopic data. Nucleic Acids Res. 32(Web Server), W668-W673. https://doi.org/10.1093/nar/gkh371 (2004).

20. Monera, O. D., Zhou, N. E., Kay, C. M. \& Hodges, R. S. Comparison of antiparallel and parallel two-stranded alpha-helical coiledcoils. Design, synthesis, and characterization. J. Biol. Chem. 268(26), 19218-19227 (1993).

21. Wood, C. W. \& Woolfson, D. N. CCBuilder 2.0: Powerful and accessible coiled-coil modeling-Powerful and accessible coiled-coil modeling. Protein Sci. 27(1), 103-111. https://doi.org/10.1002/pro.3279 (2018).

22. Franke, D. et al. ATSAS 2.8: A comprehensive data analysis suite for small-angle scattering from macromolecular solutions. J. Appl. Crystallogr. 50(4), 1212-1225. https://doi.org/10.1107/S1600576717007786 (2017).

23. Piiadov, V., Ares de Araújo, E., Oliveira Neto, M., Craievich, A. F. \& Polikarpov, I. SAXSMoW 2.0: Online calculator of the molecular weight of proteins in dilute solution from experimental SAXS data measured on a relative scale. Protein Sci. 28(2), 454-463. https:// doi.org/10.1002/pro.3528 (2019).

24. Burchardt, W. Static and dynamic light scattering approaches to structure determination of biopolymers. In Laser Light Scattering in Biochemistry 3-22 (Royal Society of Chemistry, 1992).

25. Tria, G., Mertens, H. D. T., Kachala, M. \& Svergun, D. I. Advanced ensemble modelling of flexible macromolecules using X-ray solution scattering. IUCrJ 2(2), 207-217. https://doi.org/10.1107/S205225251500202X (2015).

26. Mammen, M., Choi, S.-K. \& Whitesides, G. M. Polyvalent interactions in biological systems: Implications for design and use of multivalent ligands and inhibitors. Angew. Chem. Int. Ed. 37(20), 2754-2794. https://doi.org/10.1002/(SICI)1521-3773(19981102) 37:20<2754::AID-ANIE2754>3.0.CO;2-3 (1998).

27. Sorensen, C. S., Jendroszek, A. \& Kjaergaard, M. Linker dependence of avidity in multivalent interactions between disordered proteins. bioRxiv https://doi.org/10.1101/625327 (2019).

28. Shaw, A. et al. Binding to nanopatterned antigens is dominated by the spatial tolerance of antibodies. Nat. Nanotechnol. 14(2), 184-190. https://doi.org/10.1038/s41565-018-0336-3 (2019). 
29. Zhang, P. et al. Capturing transient antibody conformations with DNA origami epitopes. Nat. Commun. 11(1), 3114. https://doi. org/10.1038/s41467-020-16949-4 (2020).

30. Zhou, H.-X. Quantitative account of the enhanced affinity of two linked ScFvs specific for different epitopes on the same antigen. J. Mol. Biol. 329(1), 1-8. https://doi.org/10.1016/S0022-2836(03)00372-3 (2003).

31. Dill, K. A. Additivity principles in biochemistry. J. Biol. Chem. 272(2), 701-704. https://doi.org/10.1074/jbc.272.2.701 (1997).

32. Wolgemuth, C. W. \& Sun, S. X. Elasticity of $\alpha$-helical coiled coils. Phys. Rev. Lett. 97(24), 248101. https://doi.org/10.1103/PhysR evLett.97.248101 (2006).

33. Studier, F. W. Protein production by auto-induction in high-density shaking cultures. Protein Expr. Purif. 41(1), 207-234. https:// doi.org/10.1016/j.pep.2005.01.016 (2005).

34. Hoffmann, S. V., Fano, M. \& van de Weert, M. Circular dichroism spectroscopy for structural characterization of proteins. In Analytical Techniques in the Pharmaceutical Sciences (eds Müllertz, A. et al.) 223-251 (Springer, 2016). https://doi.org/10.1007/ 978-1-4939-4029-5_6.

35. Savitzky, A. \& Golay, M. J. E. Smoothing and differentiation of data by simplified least squares procedures. Anal. Chem. 36(8), 1627-1639. https://doi.org/10.1021/ac60214a047 (1964).

36. Blanchet, C. E. et al. Versatile sample environments and automation for biological solution X-ray scattering experiments at the P12 Beamline (PETRA III, DESY). J. Appl. Crystallogr. 48(2), 431-443. https://doi.org/10.1107/S160057671500254X (2015).

37. Franke, D. \& Svergun, D. I. DAMMIF, a program for rapid ab-initio shape determination in small-angle scattering. J. Appl. Crystallogr. 42(2), 342-346. https://doi.org/10.1107/S0021889809000338 (2009).

38. Volkov, V. V. \& Svergun, D. I. Uniqueness of ab initio shape determination in small-angle scattering. J. Appl. Crystallogr. 36(3), 860-864. https://doi.org/10.1107/S0021889803000268 (2003).

39. Svergun, D. I. Restoring low resolution structure of biological macromolecules from solution scattering using simulated annealing. Biophys. J. 76(6), 2879-2886. https://doi.org/10.1016/S0006-3495(99)77443-6 (1999).

40. Johnson, K. A. KinTek Global Kinetic Explorer Manual 7.0. (Kintek Corp., 2017).

41. Boal, D. H. Mechanics of the Cell 2nd edn. (Cambridge University Press, 2002).

\section{Acknowledgements}

We would like to thank Cy M. Jeffries for the assistance in using the Petra III beamline, Nykola Jones for assistance in SR-CD experiments, and Michael Ploug and Gregers R. Andersen for critical comments to the manuscript. This work was funded by a grants from the Villum Young Investigator program, The Independent Research Fund Denmark and the Marie Curie COFUND program. Access to the SR-CD beam line was granted by ISA, Centre for Storage Ring Facilities, Aarhus at Aarhus University.

\section{Author contributions}

A.J. performed all experiments. A.J. and M.K. designed the project, analysed the data and wrote the paper.

\section{Funding}

Funding was provided by Independent Research Fund Denmark - (DFF-FTP) (Grant No. 7017-00024B) and Villum Fonden (Grant No. 13165).

\section{Competing interests}

The authors declare no competing interests.

\section{Additional information}

Supplementary Information The online version contains supplementary material available at https://doi.org/ 10.1038/s41598-021-92280-2.

Correspondence and requests for materials should be addressed to M.K.

Reprints and permissions information is available at www.nature.com/reprints.

Publisher's note Springer Nature remains neutral with regard to jurisdictional claims in published maps and institutional affiliations.

(c) (i) Open Access This article is licensed under a Creative Commons Attribution 4.0 International cc) License, which permits use, sharing, adaptation, distribution and reproduction in any medium or format, as long as you give appropriate credit to the original author(s) and the source, provide a link to the Creative Commons licence, and indicate if changes were made. The images or other third party material in this article are included in the article's Creative Commons licence, unless indicated otherwise in a credit line to the material. If material is not included in the article's Creative Commons licence and your intended use is not permitted by statutory regulation or exceeds the permitted use, you will need to obtain permission directly from the copyright holder. To view a copy of this licence, visit http://creativecommons.org/licenses/by/4.0/.

(C) The Author(s) 2021 